\title{
A Novel Similarity Measure between Generalized Fuzzy Numbers
}

\author{
V. Lakshmana Gomathi Nayagam and Geetha Sivaraman
}

\begin{abstract}
In this paper, a new method has been defined to measure the degree of similarity between generalized fuzzy numbers and some properties of the proposed similarity measure have been discussed with examples. The proposed similarity measure is more accurate, robust, free from defect and precise due to the reason that the proposed similarity measure includes minimal parameters to define a generalized fuzzy numbers. This novel similarity measure has many applications in the fields of Clustering, Pattern recognition, Finger-Print matching, Fuzzy risk analysis, Decision- making and Image processing.
\end{abstract}

Index Terms-Fuzzy numbers, similarity measure, generalized fuzzy numbers.

\section{INTRODUCTION}

Zadeh introduced a concept of fuzzy sets, which has been a mathematical model to solve with imprecision [1]. The concept of similarity is fundamentally important in almost every scientific field. Degree of similarity measures of fuzzy numbers is very important in fuzzy decision making, fuzzy clustering, fuzzy risk analysis, image processing, pattern recognition [2], [3], [4] - [9], [10] - [12]. Some researchers have dealt with similarity measures for generalized fuzzy numbers in [3], [13], [14], [15], [16], [17]. All the similarity measures defined for generalized fuzzy numbers faces some drawback and fail to provide accurate result in some cases. In this paper, a new similarity measure has been proposed for generalized fuzzy numbers which includes minimal parameters to define a fuzzy number and hence it is accurate with less time.

This paper is organized as follows. Section 2 briefly reviews the definition of generalized fuzzy number and the existing similarity measure defined in [12]. Section 3 provides the new similarity measure between the generalized fuzzy numbers, some of its properties and comparison of the proposed measure with existing measure. In section 4, conclusions are drawn.

\section{PRELIMINARIES}

Here we give a brief review of preliminaries.

A generalized fuzzy number $A=(a, b, c, d, w)$, where

Manuscript received March 8, 2012; revised May 10, 2012.

V. Lakshmana Gomathi Nayagam is with the Department of Mathematics, National Institute of Technology, Tiruchirappalli, Tamil Nadu, India (e-mail: velulakshmanan@nitt.edu).

Geetha Sivaraman is with the PI(WOS-A), DST India, Department of Mathematics, National Institute of Technology, Tiruchirappalli, Tamil Nadu, India (e-mail: geedhasivaraman@ yahoo.com).
$0 \leq a \leq b \leq c \leq d \leq 1$ and $0 \leq w \leq 1$, is a fuzzy subset of the real line $\mathrm{R}$ with membership function $\mu_{A}$ which has the following properties [9].

(a) $\mu_{A}$ is a continuous mapping from $\mathrm{R}$ to the closed interval $[0, w], \quad 0 \leq w \leq 1$.

(b) $\mu_{A}(x)=0$ for all $x \in(-\infty, a]$.

(c) $\mu_{A}$ is strictly increasing on $[a, b]$.

(d) $\mu_{A}(x)=w$ for all $x \in[b, c]$, where $w$ is a constant and $0 \leq w \leq 1$.

(e) $\mu_{A}$ is strictly decreasing on $[c, d]$.

(f) $\mu_{A}(x)=0$ for all $x \in[d,+\infty)$.

In a generalized fuzzy number, $\mu_{A}$ is linear in $[a, b]$ and $[c, d$,$] then it is called generalized trapezoidal fuzzy$ number.

Wei and Chen defined a new approach for similarity measures of generalized fuzzy numbers and compared with existing similarity measures [12].

Let $\quad A=\left(a_{1}, a_{2}, a_{3}, a_{4}, w_{A}\right)$ and $B=\left(b_{1}, b_{2}, b_{3}, b_{4}, w_{B}\right)$ be two generalized trapezoidal fuzzy numbers. Then the degree of similarity $S(A, B)$ between the generalized trapezoidal fuzzy numbers $A$ and $B$ can be calculated as follows:

$$
\begin{aligned}
& S(A, B)=\left(1-\frac{\sum_{i=1}^{4}\left|a_{i}-b_{i}\right|}{4}\right) \times \\
& \frac{\min (P(A), P(B))+\min \left(w_{A}, w_{B}\right)}{\max (P(A), P(B))+\max \left(w_{A}, w_{B}\right)}
\end{aligned}
$$

where $P(A)$ and $P(B)$, the perimeters of the generalized trapezoidal fuzzy numbers $A$ and $B$ respectively are defined as follows:

$$
\begin{gathered}
P(A)=\sqrt{\left(a_{1}-a_{2}\right)^{2}+w_{A}^{2}}+\sqrt{\left(a_{3}-a_{4}\right)^{2}+w_{A}^{2}}+ \\
\left(a_{3}-a_{2}\right)+\left(a_{4}-a_{1}\right) \\
P(B)=\sqrt{\left(b_{1}-b_{2}\right)^{2}+w_{B}^{2}}+\sqrt{\left(b_{3}-b_{4}\right)^{2}+w_{B}^{2}}+ \\
\left(b_{3}-b_{2}\right)+\left(b_{4}-b_{1}\right)
\end{gathered}
$$


The larger the value of $S(A, B)$, the greater the similarity between the fuzzy numbers $A$ and $B$.

But the above defined similarity measure also fails to provide accurate results at some cases which will be dealt in further section.

\section{The Novel Similarity Measures BetweEN GENERALIZED FUZZY NUMBERS}

In this section, we present a new method for the degree of similarity between generalized fuzzy numbers and prove some properties of the proposed similarity measure.

$$
\text { Let } \quad A=\left(a_{1}, a_{2}, a_{3}, a_{4}, w_{A}\right) \quad \text { and }
$$

$B=\left(b_{1}, b_{2}, b_{3}, b_{4}, w_{B}\right)$ be two generalized trapezoidal fuzzy numbers. Then the degree of similarity $S(A, B)$ between the generalized trapezoidal fuzzy numbers $A$ and $B$ is given by

$$
\begin{aligned}
& S(A, B)=\left[\sqrt[4]{\prod_{i=1}^{4}\left[1-\left|a_{i}-b_{i}\right|\right]}\right] \times \\
& \frac{\operatorname{Min}(P(A), P(B))+\operatorname{Min}\left(w_{A}, w_{B}\right)}{\operatorname{Max}(P(A), P(B))+\operatorname{Max}\left(w_{A}, w_{B}\right)}
\end{aligned}
$$

where $P(A)$ and $P(B)$ are

$$
\begin{gathered}
P(A)=\sqrt{\left(a_{2}-a_{1}\right)^{2}+\left(a_{3}-a_{2}\right)^{2}+\left(a_{4}-a_{3}\right)^{2}+w_{A}^{2}} \\
P(B)=\sqrt{\left(b_{2}-b_{1}\right)^{2}+\left(b_{3}-b_{2}\right)^{2}+\left(b_{4}-b_{3}\right)^{2}+w_{B}^{2}}
\end{gathered}
$$

Clearly $P(A)$ and $P(B)$ are the minimum distances of the generalized trapezoidal fuzzy number $A$ and $B$ respectively which includes height and scales of generalized fuzzy number. The larger the value of $S(A, B)$, the greater the similarity between the fuzzy numbers $A$ and $B$.

\section{A. Properties of Proposed Similarity Measure}

Property 1: The similarity measure of the two generalized trapezoidal fuzzy number must lie between 0 and 1 i.e. $0 \leq S(A, B) \leq 1$.

Property 2: Two generalized trapezoidal fuzzy numbers $A$ and $B$ are identical if and only if $S(A, B)=1$.

Property 3: $S(A, B)=S(B, A)$.

Property 4: If $A=(a, a, a, a, 1)$ and $B=(b, b, b, b, 1)$ are two real numbers, then $S(A, B)=1-|a-b|$.

\section{B. Drawbacks of the Existing Similarity Measure}

1) Consider the generalized fuzzy numbers $A=(0.1,0.2,0.3,0.4,0)$ and $B=(0.1,0.2,0.3,0.4,1.0)$, where $w_{A}=0$ and $w_{B}=1.0$ as in the following figure. By equation 1 , the similarity measure between $A$ and $B(=0.1759)$ is more when compared to the similarity measure between
$A$ and $B(=0.086)$ using proposed method.

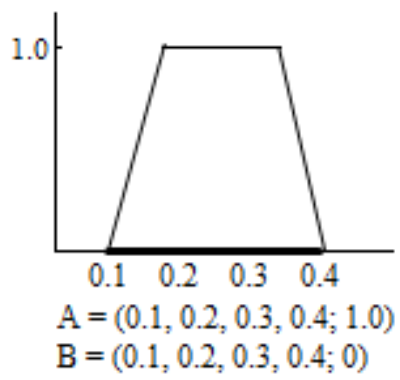

2) Consider the generalized fuzzy numbers $A$ and $B$ where $\quad A=(0.01,0.01,0.01,0.01,0.2)$ and $B=(0.89,0.90,0.94,0.95,1.0)$. Since the generalized fuzzy numbers $A$ and $B$ are entirely different the similarity measure between $A$ and $B$ are definitely less. But the proposed method also gives the accurate degree of similarity between $A$ and $B$ $(=0.017)$ when it is compared with degree of similarity between $A$ and $B(=0.176)$ by equation 1 .

3) Consider the generalized fuzzy numbers $A=(0.1,0.1,0.2,0.3,1)$ and

$B=(0.4,0.4,0.6,0.6,1.0)$,

$C=(0.1,0.1,0.3,0.5,1)$ and

$D=(0.45,0.45,0.65,0.85,1.0)$. By equation 1 , we yield $\mathrm{S}(\mathrm{A}, \mathrm{B})=\mathrm{S}(\mathrm{C}, \mathrm{D})=0.65$ which is illogical. But by the proposed method we get $S(A, B)=0.67 \neq 0.65$.

4) Consider the generalized fuzzy numbers $A=(0.1,0.1,0.2,0.3,1)$ and

$B=(0.2,0.3,0.4,0.4,1.0)$,

$C=(0.1,0.2,0.3,0.4,1)$

and

$D=(0.25,0.35,0.45,0.55,1.0)$. By equation 1 , we yield $S(A, B)=S(C, D)=0.85$ which is anti-intuitive to $\mathrm{S}(\mathrm{C}, \mathrm{D})=0.85>\mathrm{S}(\mathrm{A}, \mathrm{B})=0.8485$.

5) Consider $A=(0,0.1,0.4,0.5,1)$ $B=(0.1,0.2,0.3,0.4,1) \quad C=(0,0.1,0.4,0.5,1)$ and $D=(0.2,0.3,0.4,0.5,1.0)$. By equation 1 , we get $\mathrm{S}(\mathrm{A}, \mathrm{B})=\mathrm{S}(\mathrm{C}, \mathrm{D})$ which is anti-intuitive to $\mathrm{S}(\mathrm{C}, \mathrm{D})=$ $0.887<\mathrm{S}(\mathrm{A}, \mathrm{B})=0.883$.

\section{Importance of the Proposed Similarity Measure}

Wei and Chen defined a similarity measure of generalized fuzzy numbers which is the function of $a, b, c, d$, and $w$ which are the parameters of generalized fuzzy numbers [12]. But the proposed similarity measure is again smaller function of $a, b$, $c, d$, and $w$ which includes height and all scales of generalized fuzzy number by excluding repetition. So this proposed measure obviously reduces the running time of the programme.

\section{CONCLUSION}

In this paper, a new similarity measure between generalized fuzzy numbers has been defined by modifying 
the method of Wei and Chen by excluding the repetition of parameters [12]. So it reduces the time complexity and some of drawbacks of the existing similarity measure are being overruled by the proposed measure.

\section{REFERENCES}

[1] L. A. Zadeh, "Fuzzy Sets," Information and Control, vol. 8, pp. $338-$ 353, 1965.

[2] D. V. Weken, M. Nachtegael, and E. E. Kerre, "Using similarity measures and homogeneity for the comparison of images," Image and Vision Computing, vol. 22, pp. 695 - 702, 2004.

[3] İ. Güler and E. D. Übeyli, "Analysis of Doppler ultrasound signals based on fuzzy similarity index," Expert Systems with Applications, vol. 31, pp. $130-136,2006$.

[4] M. S. Yang and D. C. Lin, "On similarity and inclusion measures between type-2 fuzzy sets with an application to clustering," Computers \& Mathematics with Applications, vol. 57, pp. 896 - 907 , 2009.

[5] H. B. Mitchell, "On the Dengfeng-Chuntian similarity measure and its application to pattern recognition," Pattern Recognition Letters, vol. 24, pp. $3101-3104,2003$.

[6] N. Karacapilidis and C. Pappis, "Computer-supported collaborative argumentation and fuzzy similarity measures in multiple criteria decision making," Computers \& Operations Research, vol. 27, pp. 653 $-671,2000$.

[7] B. Prados-Suárez, J. Chamorro-Martínez, D. Sánchez, and J. Abad, "Region-based fit of color homogeneity measures for fuzzy image segmentation," Fuzzy Sets and Systems, vol. 158, pp. 215 - 229, 2007.

[8] R. Saraçoğlu, K. Tütüncü, and N. Allahverdi, "A fuzzy clustering approach for finding similar documents using a novel similarity measure," Experts Systems with Applications, vol. 33, pp. 600 - 605, 2007.

[9] S. J. Chen and S. M. Chen, "Fuzzy risk analysis based on similarity measures of generalized fuzzy numbers," IEEE Transactions on Fuzzy systems, vol. 11 , pp. $45-56,2003$.

[10] S. M. Chen, "New methods for subjective mental workload assessment and fuzzy risk analysis," Cybernetics and Systems: An International Journal, vol. 27, pp. 449-472, 2001.

[11] T. Gu and B. Dubuisson, "Similarity of classes and fuzzy clustering," Fuzzy Sets and Systems, vol. 34, pp. 213 - 221, 1990.

[12] S. H. Wei and S. M. Chen, "A new approach for fuzzy risk analysis based on similarity measures of generalized fuzzy numbers," Expert Systems with Applications, vol. 36, pp. 589 - 598, 2009.

[13] C. H. Hsieh and S. H. Chen, "Similarity of generalized fuzzy numbers with graded mean integration representation," in Proceedings of the Eighth International Fuzzy Systems Association World Congress, vol. 2, pp. $551-555,1999$.

[14] H. S. Lee, "An optimal aggregation method for fuzzy opinions of group decision," Proceedings of the 1999 IEEE International conference on Systems, Man and Cybernetics, vol. 3, pp. 314 - 319, 1999.

[15] S. J. Chen, "A new similarity measure of generalized fuzzy numbers based on Geometric-Mean Averaging operator," in Proceedings of the IEEE International Conference on Fuzzy Systems, pp. 1879 - 1886, Fuzz-IEEE 2006, Vancouver, Canada, 2006.

[16] S. J. Chen, "Similarity measure between Generalized Fuzzy Numbers using Quadratic- Mean operator," Second International Symposium on Intelligent Information Technology Application, vol. 3, pp. 440 - 444, 2008.

[17] D. Yong, S. Wenkang, D. Feng, and L. Qi, “A new similarity measure of generalized fuzzy numbers and its application to pattern recognition," Pattern Recognition Letters, vol. 25, pp. 875 - 883, 2004. 